\title{
Infrared Imaging Systems for wall protection in the W7-X stellarator (invited) ${ }^{a}$
}

Marcin Jakubowski, ${ }^{1,2}$ Peter Drewelow, ${ }^{1}$ Joris Fellinger, ${ }^{1}$ Aleix Puig Sitjes, ${ }^{1}$ Glen Wurden, ${ }^{3}$ Adnan Ali, ${ }^{1}$ Christoph Biedermann, ${ }^{1}$ Barbara Cannas, ${ }^{4}$ Didier Chauvin, ${ }^{1}$ Marc Gamradt, ${ }^{1}$ Henry Greve, ${ }^{1}$ Yu Gao, ${ }^{5}$ Dag Hathiramani, ${ }^{1}$ Ralf König, ${ }^{1}$ Axel Lorenz, ${ }^{1}$ Victor Moncada, ${ }^{6}$ Holger Niemann, ${ }^{1}$ Tran Thanh Ngo, ${ }^{7}$ Fabio Pisano, ${ }^{4}$ Thomas Sunn Pedersen, ${ }^{1}$ and W7-X team ${ }^{\mathrm{b})}$

1) Max-Planck-Institut für Plasmaphysik, 17491 Greifswald, Germany

${ }^{2)}$ University of Szczecin, Institute of Physics, 70-451 Szczecin, Poland

3) Los Alamos National Laboratory, NM 87545 Los Alamos, $U S A$

4) University of Cagliari, 09124 Cagliari, Italy

5) Forschungszentrum Jülich GmbH, IEK-4, 52428 Jülich, Germany

6) Thermadiag, 13590 Meyreul, France

7) CEA Cadarache, 13108 Cadarache, France

(Dated: 23 November 2018) 
Wendelstein 7-X aims at quasi-steady state operation with up to $10 \mathrm{MW}$ of heating power for 30 minutes. Power exhaust will be handled predominantly via 10 actively water cooled CFC based divertor units designed to withstand power loads of $10 \mathrm{MW} / \mathrm{m}^{2}$ locally in steady state. If local loads exceed this value, a risk of local delamination of the $\mathrm{CFC}$ and failure of entire divertor modules arises. Infrared endoscopes to monitor all main plasma facing components are being prepared and near real time software tools are under development to identify areas of excessive temperature rise, to distinguish them from non-critical events, and to trigger alarms. Tests with different cameras were made in the recent campaign. Long pulse operation enforces additional diagnostics design constraints: e.g. the optics need to be thermally decoupled from the endoscope housing. In the upcoming experimental campaign a graphite scraper element (SE), in front of the island divertor throat will be tested as a possible means to protect the divertor pumping gap edges during the transient discharge evolution.

\footnotetext{
a) Paper published as part of the Proceedings of the 22nd Topical Conference on High-Temperature Plasma Diagnostics, San Diego, California, April, 2018

b) All members of W7-X team are listed in R. Wolf, et al., ${ }^{1}$
} 


\section{WENDELSTEIN 7-X}

As fusion experiments progress towards steady state operation, plasma facing components are undergoing a significant change in their design. Namely there is a need for transition from inertially cooled pulsed systems to steady state designs with significant power handling capacity. The power loads must stay below the engineering limits set by material constraints (e.g. due to erosion or melting of the plasma facing component) ${ }^{2}$. Wendelstein 7-X, an advanced superconducting stellarator in Greifswald, Germany aims to tackle the issues of steady state plasma wall interactions at ITER relevant power loads. The main heating system at W7-X is Electron Cyclotron Resonance Heating (ECRH), which uses microwave power of up to $8 \mathrm{MW}$ in continuous wave operation at a frequency of $140 \mathrm{GHz}^{3}$. This allows for discharges of up to 30 minutes duration, which requires a rather sophisticated power handling concept. As W7-X operates in a non-axisymmetric configuration with a helix-like magnetic axis and five identical magnetic field periods an open divertor structure has been chosen, which benefits from the inherent divertor property of the magnetic configuration. The so called island divertor utilizes natural magnetic island chains forming at the plasma boundary (in most cases at edge iota $t=1$ ) that are intersected by divertor target plates. This concept was for the first time successfully tested in $\mathrm{W} 7-\mathrm{AS}^{4}$. The system will allow an effective particle and energy exhaust for a wide range of plasma and magnetic parameters. Very important for the fusion experiment operating in quasi steady state is the durability of the plasma facing components (PFC). At W7-X rather different loads on the PFC are expected, e.g. the central wall protection system and the ports are expected to get ca. 100 $\mathrm{kW} / \mathrm{m}^{2}$, while the divertor should be able to cope with $10 \mathrm{MW} / \mathrm{m}^{2}$ for 30 minutes and even higher transient loads. Various design and technological solutions have been developed at W7-X to meet these high load requirements in the complicated 3D-shaped geometry of the plasma vessel ${ }^{5}$.

High-power steady-state operation of W7-X is approached in three steps (see Table I) in order to allow for careful tests of its components and key diagnostics.

- In the first operational phase (OP1.1) most of the graphite armor components and the island divertor were not installed. To protect metal parts, in particular at that time the unprotected divertor frame structure and to guarantee reasonable performance, a so-called limiter configuration was used ${ }^{6}$. Five graphite limiters, matching the five-fold 


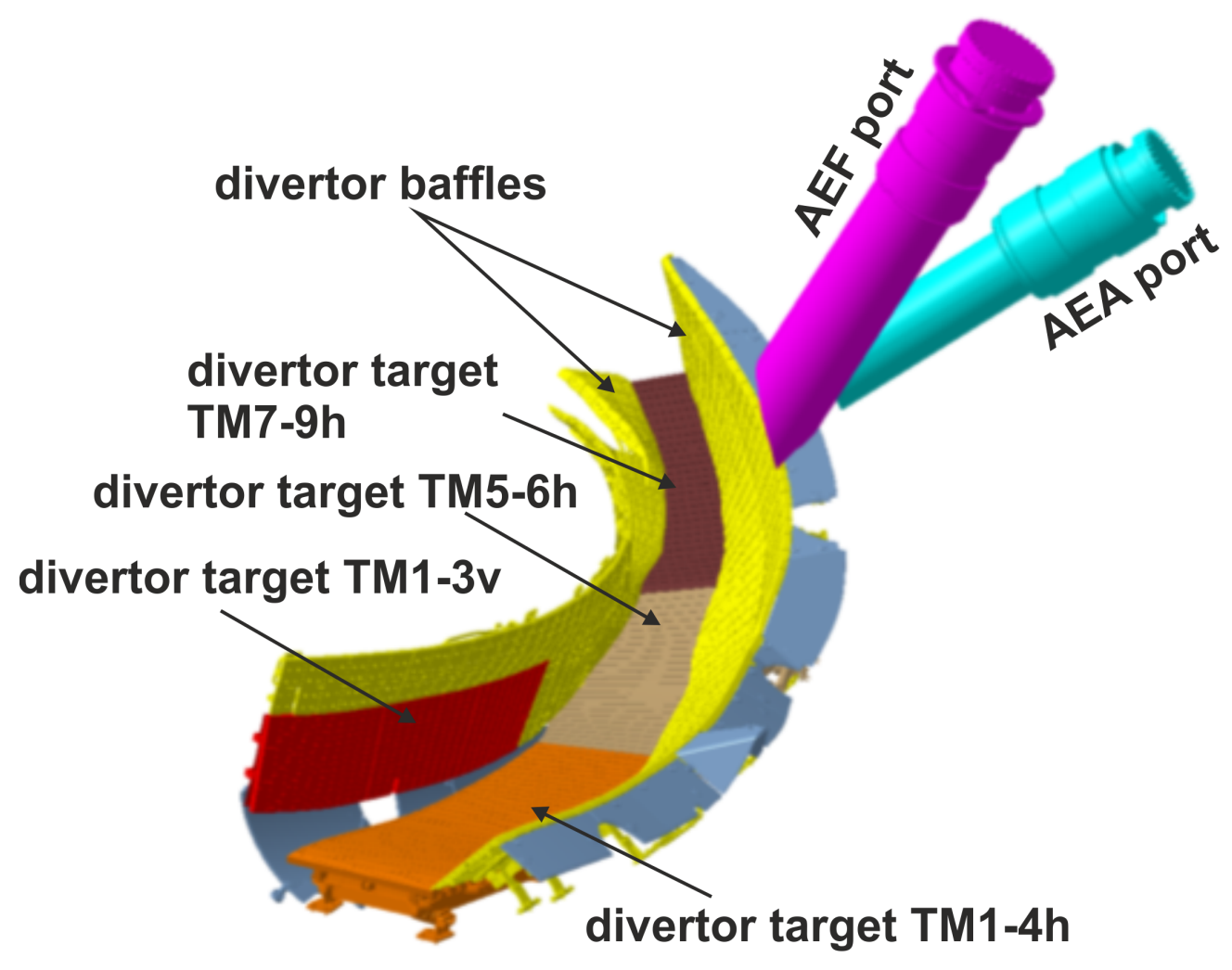

FIG. 1. One out of $10 \mathrm{~W} 7-\mathrm{X}$ divertors. Each one consists of horizontal target modules(TM1-4h, TM5-6h and TM7-9h), vertical target modules (TM1-3v) and divertor baffles. Depending on the magnetic configuration different parts of the divertor are receiving power loads from plasma edge. Target modules TM1-4h, TM1-3v form a so-called standard divertor region and TM7-9h a so-called high iota divertor. Both names refer to magnetic configurations, which put loads on those parts of the W7-X divertor. In the present campaign (OP1.2) the divertor is inertially cooled. From 2020 (OP2)it will be water-cooled. The observation with IR cameras is performed mostly through ca. $2 \mathrm{~m}$ long port tubes (labeled AEF and AEA)

symmetry of the plasma, were installed in symmetry planes at the inboard side. The magnetic vacuum configuration has been chosen such that it has a smooth scrapeoff layer, with no stochastic region and no large magnetic islands. Thus, the limiters efficiently intercepted $>99 \%$ of the convective plasma heat load in the SOL. It allowed for testing of many components of W7-X (including edge diagnostics ${ }^{7,8}$ ).

- In the ongoing campaign (OP1.2) the machine is fully armored with carbon plasma facing components including ten inertially cooled divertor units (see Fig. 1). During 
OP1.2 a heating power of up to $8 \mathrm{MW}$ is used with discharge duration given by limits allowed to test the divertor performance. This required an upgrade of many diagnostics in order to cope with longer pulse duration and higher input power ${ }^{9}$. The divertor shape is identical to the water-cooled divertor prepared for the next campaign.

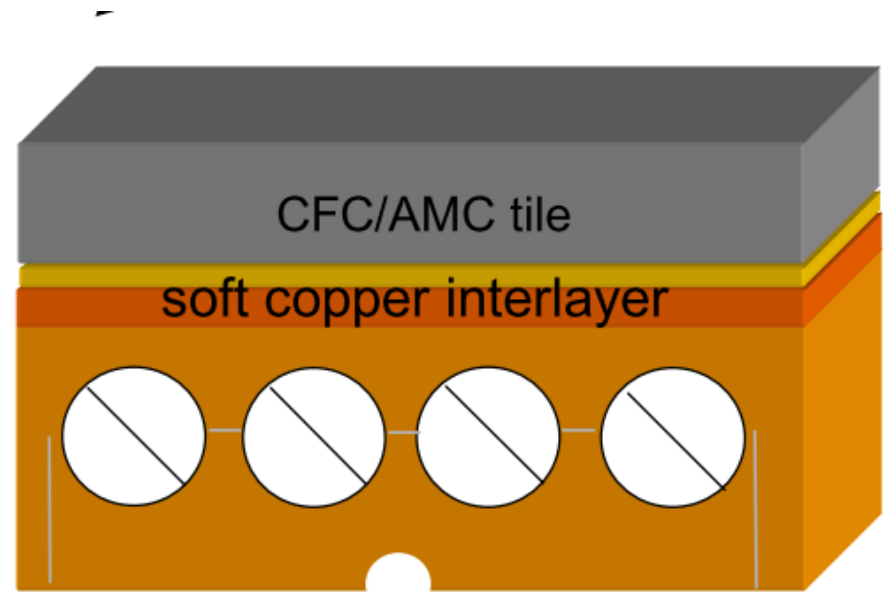

(a)

(b)

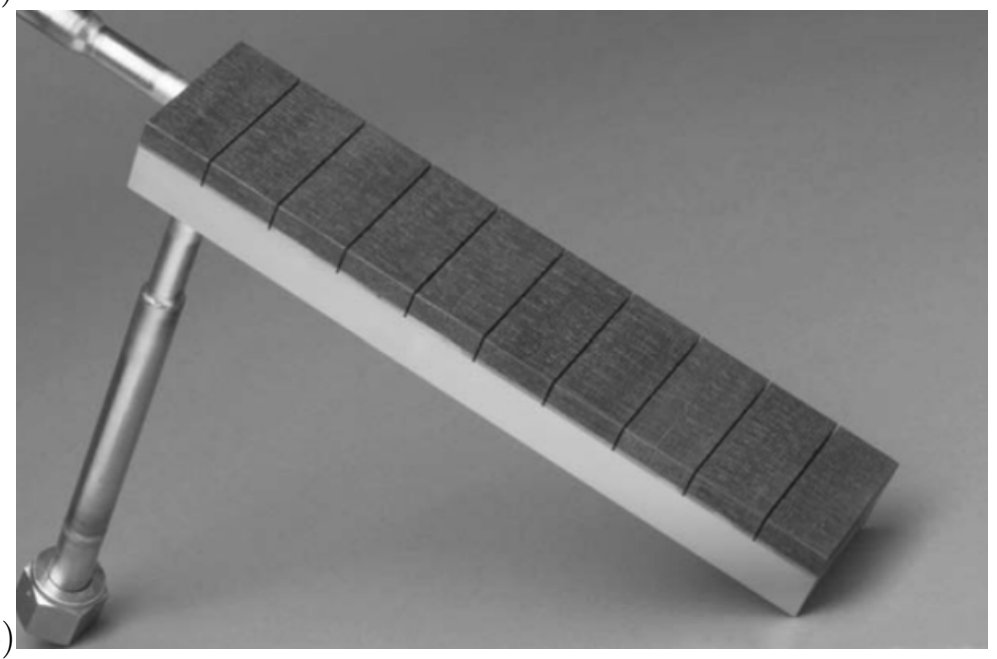

FIG. 2. (a) Sketch presenting the structure of one of the high heat flux target elements and (b) the actual image of a target element.

- From 2020 (OP2) the divertors at Wendelstein 7-X (see Fig. 1) will be composed of high heat load target tiles, which can sustain up to $10 \mathrm{MW} / \mathrm{m}^{2}$ and a lower heat load baffle structure designed for up to $0.5 \mathrm{MW} / \mathrm{m}^{2}$ of continuous power loads ${ }^{5,10}$. Figure 2 presents a sketch of the internal structure of divertor target plates. The high heat load targets consist of up to $500 \mathrm{~mm}$ long and $50 \mathrm{~mm}$ wide individual target elements (Fig. 2(b)) which are covered with an on average $6 \mathrm{~mm}$ thick CFC top layer (marked as 
gray in Fig. 2(a)) structured as 25x50 mm individual blocks. They are connected via an AMC-copper interlayer (yellow and dark orange) to an underlying CuCrZr cooling block (orange), which contains 4 cooling water channels. To maintain the functionality of the target plates the interlayer may not exceed temperatures of $475{ }^{\circ} \mathrm{C}^{11}$. If local temperatures exceed this value, the resulting stress can lead to delamination of the CFC-Cu layer and eventually failure of these components. Because of this limitation, a close control of the target tile temperatures is of utmost importance.

\section{INFRARED DIAGNOSTICS}

The possible disadvantage of an island divertor is its sensitivity to external error fields and inaccuracies in installation of plasma-facing components. In steady state operation, assumptions that power loads follow the symmetry of the device cannot be made without testing it first. Monitoring of the temperature of individual components through the use of thermocouples is impossible due to the presence of the cooling water channels. Instead, the divertor surface temperature will be measured through infrared light observation. Ten highresolution infrared systems are installed to monitor the whole divertor surface consisting of 5 upper and 5 lower divertors (each equipped with a horizontal and vertical target). Based on the temperature evolution, the heat flux from the plasma is derived using a two-dimensional thermal model (THEODOR ${ }^{12}$ ). The staged approach to high performance discharges allows us to develop the infrared diagnostics in several steps.

\section{A. Limiter observation}

Several cameras have been installed before OP1.1 to observe the limiters and the inner wall throughout the campaign, among them infrared cameras to measure temperature distribution on the limiter surfaces. Three types of IR cameras have been used. Nine NIR cameras (SONY XC-EI50CE with a filter i 0.8- $\mu \mathrm{m}$ ) and one LWIR camera (DIAS Pyroview measuring at 10-14 $\mu \mathrm{m}$ ), viewing the limiter in module 5). The nine systems with NIR and the system with LWIR camera were placed following W7-X symmetry in all AEF ports. The data from NIR cameras suffered from plasma emission near the limiter surface. As they had low dynamic range $(8$ bit) they offered a rather limited temperature range and were set to 


\begin{tabular}{ccccc}
\hline campaign & main & $E_{\text {max }}$ & $t_{\text {max }}$ & $T_{\text {max }}$ \\
(years) & PFC & {$[\mathrm{MJ}]$} & {$[\mathrm{s}]$} & {$\left[{ }^{\circ} \mathrm{C}\right]$} \\
\hline OP1.1 & limiter & 4 & 6 & 800 \\
$(2015-6)$ & & & \\
OP1.2 & inertially & 80 & 20 & 1600 \\
$(2017-8)$ & cooled & & & \\
& divertor & & & \\
OP2 & water & & & \\
$(2020-)$ & cooled & & \\
& divertor & & \\
\hline
\end{tabular}

TABLE I. Stages of W7-X operation. The columns contain following information (from left): campaign, main plasma facing component, maximum allowed plasma energy input, maximum duration of a discharge, maximum surface temperature of the main plasma facing component

detect temperatures above $500{ }^{\circ} \mathrm{C}$.

In Fig. 3 the temperature distribution on the limiter in module 5 measured with the LWIR camera is shown. Additionally, a high-resolution MWIR camera (a 3-5 $\mu \mathrm{m}$ band infrared (FLIR SC8303HD) camera (125 Hz full-frame rate, $1344 \times 768$ pixels) and a 400800nm AVT Prosilica GX-1050C (100 Hz full-frame rate, $1024 \times 1024$ pixels) color visible camera co-located on the same line of sight. Both cameras delivered high spatial (ca. $1 \mathrm{~mm}$ ) and temporal (100-400 Hz) images of the limiter ${ }^{13}$.

\section{B. Immersion tubes}

In OP1.1. and OP1.2 the main system, which allows observation of either limiter or divertor surface, was a so called immersion tube with a design shown in Fig. 4. It is inserted into 9 out of $10 \mathrm{AEF}$-port flanges (see figure 1(b)) and it contains three cameras near the inner vacuum vessel surface.

Two visible cameras (blue) each with narrow band pass filter $\left(\mathrm{H}_{\alpha}, \mathrm{H}_{\gamma}\right.$ or carbon lines) and one infrared (LWIR) micro-bolometric camera (red) monitor the divertor surface tem- 


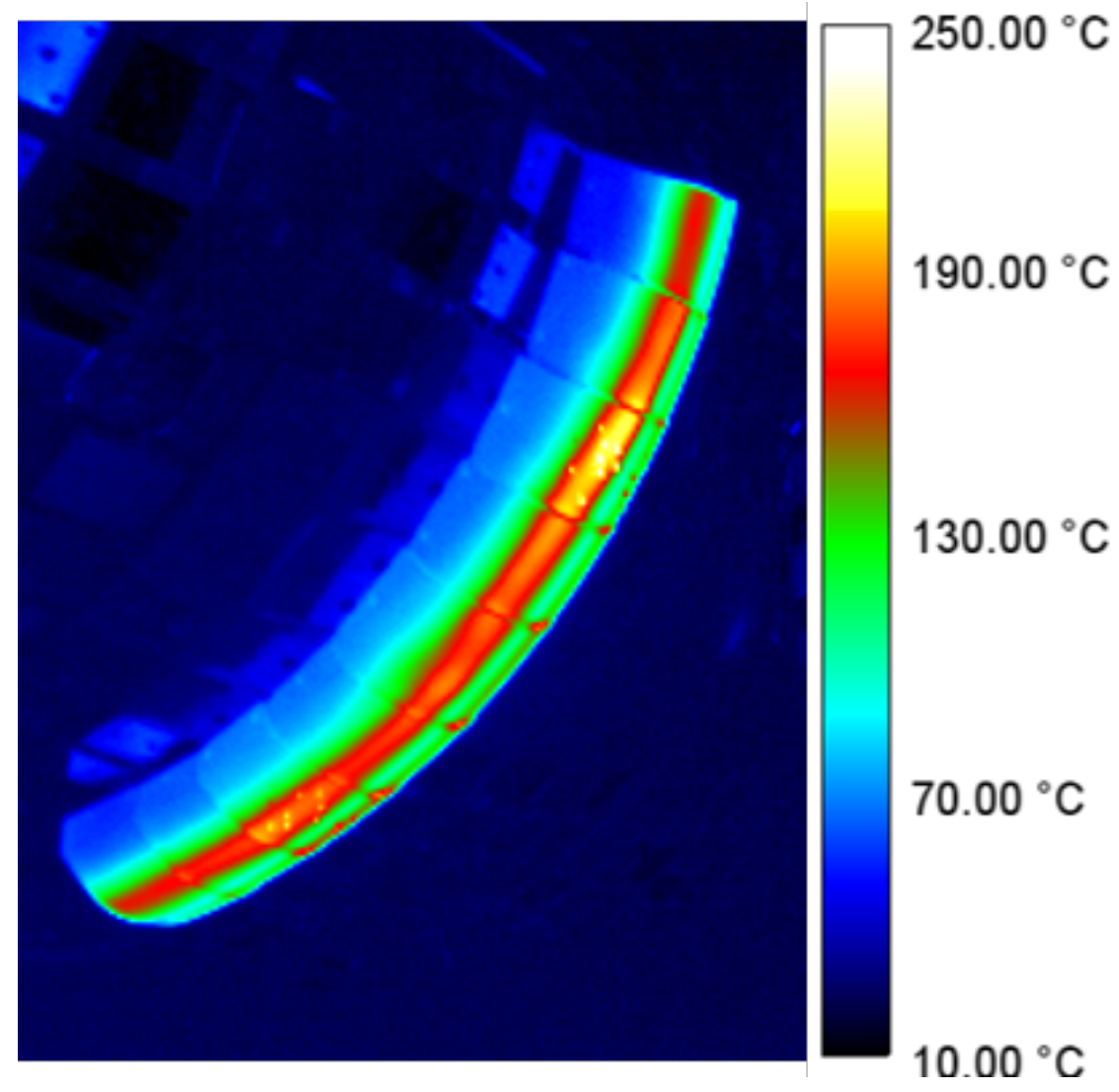

FIG. 3. View on one of the inboard limiters via LWIR camera mounted in immersion tube

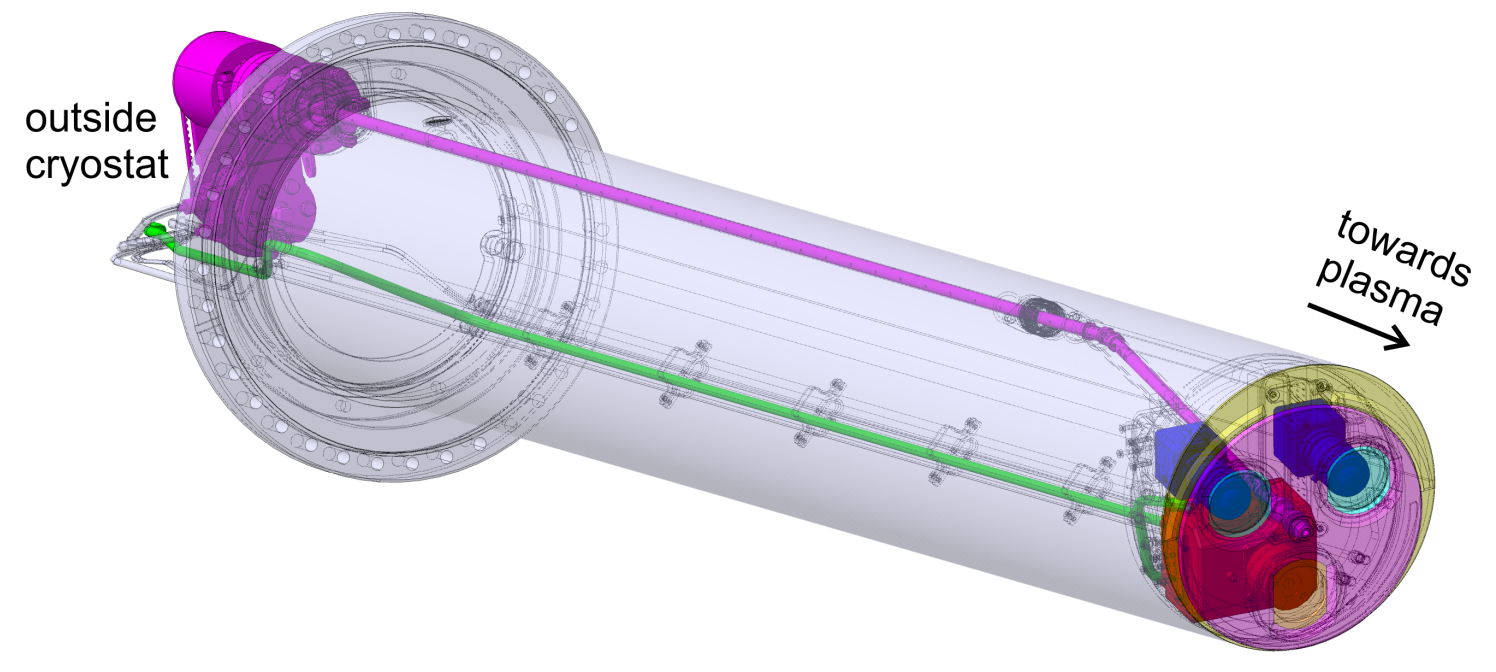

FIG. 4. Overview of immersion tube with visible cameras (blue) and infrared camera (red) behind vacuum windows (light blue and orange) in a plasma facing front plate (yellow) covered by a rotating shutter (pink) 
perature and edge light emission in a wide angle view. In OP1.2 all NIR cameras (used for limiter observation in OP1.1) were replaced by the LWIR cameras (IRcam Caleo 768k L, $1024 \times 768$ pixels, pitch $17 \mu \mathrm{m})$. These cameras have been built specifically to survive in high magnetic field $\leq 3 \mathrm{~T}$ by separating sensor from acquisition electronics. A fish eye lens provides wide angle view of the whole divertor surface $\left(\mathrm{FOV} \approx 116^{\circ}\right)$. The spectral response of the camera has been limited by a shortpass filter to $8-10 \mu \mathrm{m}$. This allows the measurement of temperatures from $30^{\circ} \mathrm{C}$ up to $5000^{\circ} \mathrm{C}$. Each pixel of the sensor is a resistive microbolometer that measures a temperature rise due to impinging radiation. Although cooled by a pressurized air flow inside the camera housing, the sensors are sensitive to temperature drifts during plasma operation. The shutter in front of the camera has been used to acquire a homogeneous reference image at ambient temperature in order to correct the temperature drifts. The shutter is operated via pressurized air (3 bar).

In addition to the LWIR camera each immersion tube contained an absolutely calibrated visible imaging cameras, which provide information about carbon generation and concentration or the hydrogen recycling conditions. For protection against surface coatings a pneumatically driven shutter (pink in Fig. 4) can be closed in front of the windows. Pressurized air-cooling (green) is necessary for cooling internal heating by the cameras and the radiative heat load during plasma operation.

In Fig. 5 an example of the temperature distribution on the divertor surface is shown. The thermal image is overlaid with a CAD model of the vessel. In order to match the CAD vessel model with the image, the CAD model projection had to be deformed to match the fish eye optical distortion of the camera ${ }^{14}$. The achievable resolution in the low iota target area is ca. $4 \mathrm{~mm}$ on horizontal target (TM1-4h) and $6 \mathrm{~mm}$ on vertical targets (TM1-3v). In the high iota area (TM7-9h) $5 \mathrm{~mm}$ can be resolved at the closer part of high iota and $9 \mathrm{~mm}$ at the far end of high iota target plates.

The main diagnostic to assure safe high heat flux divertor operation of Wendelstein 7-X starting in OP2 will be a set of 10 endoscopes monitoring the divertor and baffles surface and the plasma radiation near their surface. As each of the W7-X divertor modules consists of target elements with dimensions of $25 \mathrm{~mm}$ x $50 \mathrm{~mm}$ (see Fig. 2b) the minimum required optical resolution of the system, which allows measuring surface temperatures of individual target elements, is of the order of $5 \mathrm{~mm}$. Due to several constraints on the thermographic system (e.g. to reduce neutron loads) the cameras need to be placed 2 


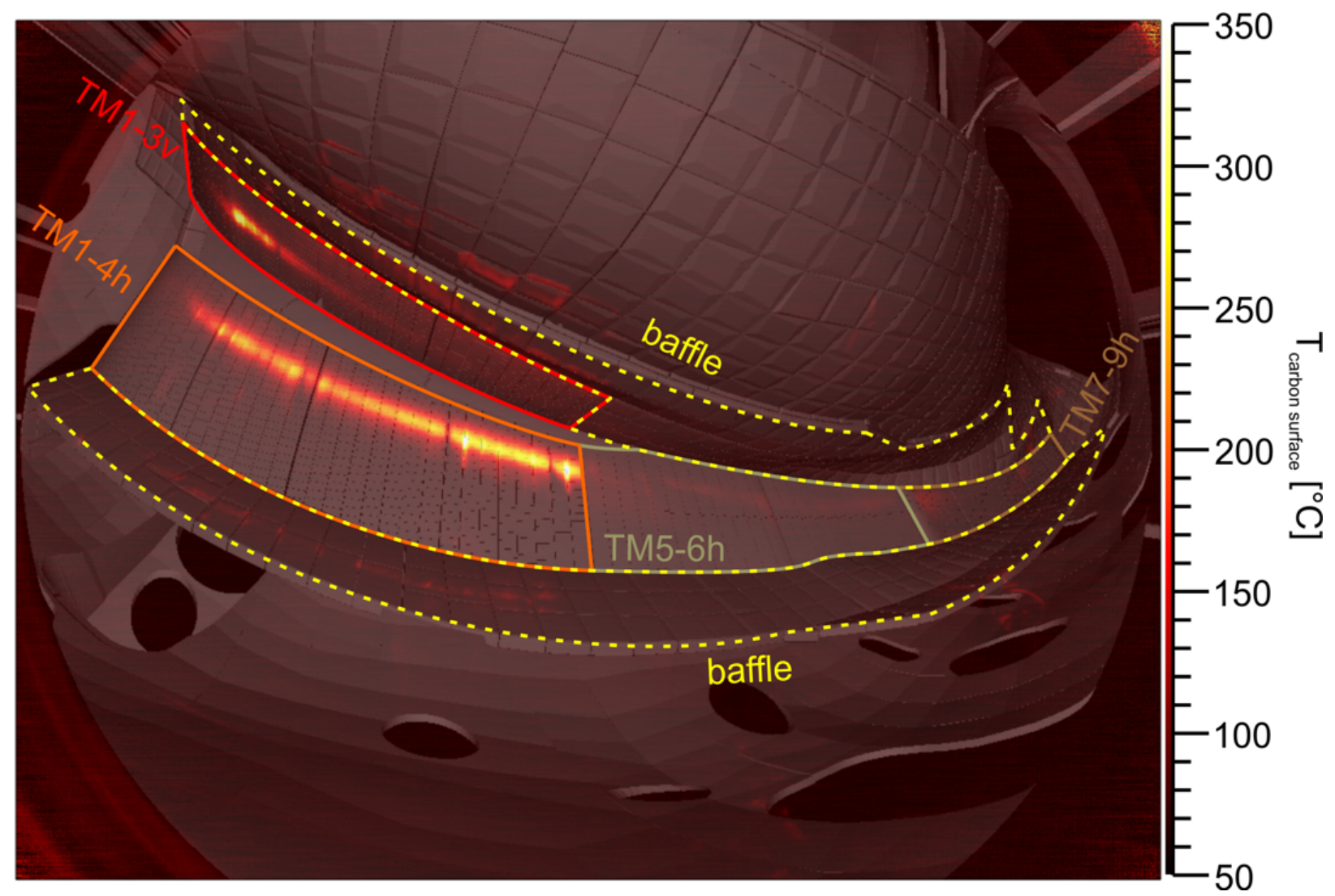

FIG. 5. An infrared view of a divertor in module 2 made by a microbolometric infrared camera inside the immersion tube. Image is overlaid with a CAD model of the vessel. Colors indicate carbon surface temperature.

$\mathrm{m}$ away from the observed surfaces and therefore use a set of optics to collect the light from the divertor surface. The advantage of InSb cameras over the LWIR micro-bolometer cameras is lower diffraction limit at a wavelength of $4 \mu \mathrm{m}$ rather than $10 \mu \mathrm{m}$ and higher pixel number on the presently available sensors. Therefore a decision has been made to develop the optics for the InSb cameras operating at ca. $4 \mu \mathrm{m}$. The endoscope cannot use entrance windows on the plasma side as during operation they would change significantly the endoscope transmission due to deposits coming from plasmas. This would make proper thermographic measurements virtually impossible. The design of the optical system is based on Cassegrain optics ${ }^{15}$ following the results of JET endoscopes ${ }^{16}$.

The OP2 endoscope development was started by commissioning a prototype for tests in OP1.2 $\mathrm{a}^{17}$. Its functional sketch is presented in Fig. 7. The light coming from a divertor enters the endoscope via a pinhole and it is transmitted via a complex system of mirrors and 


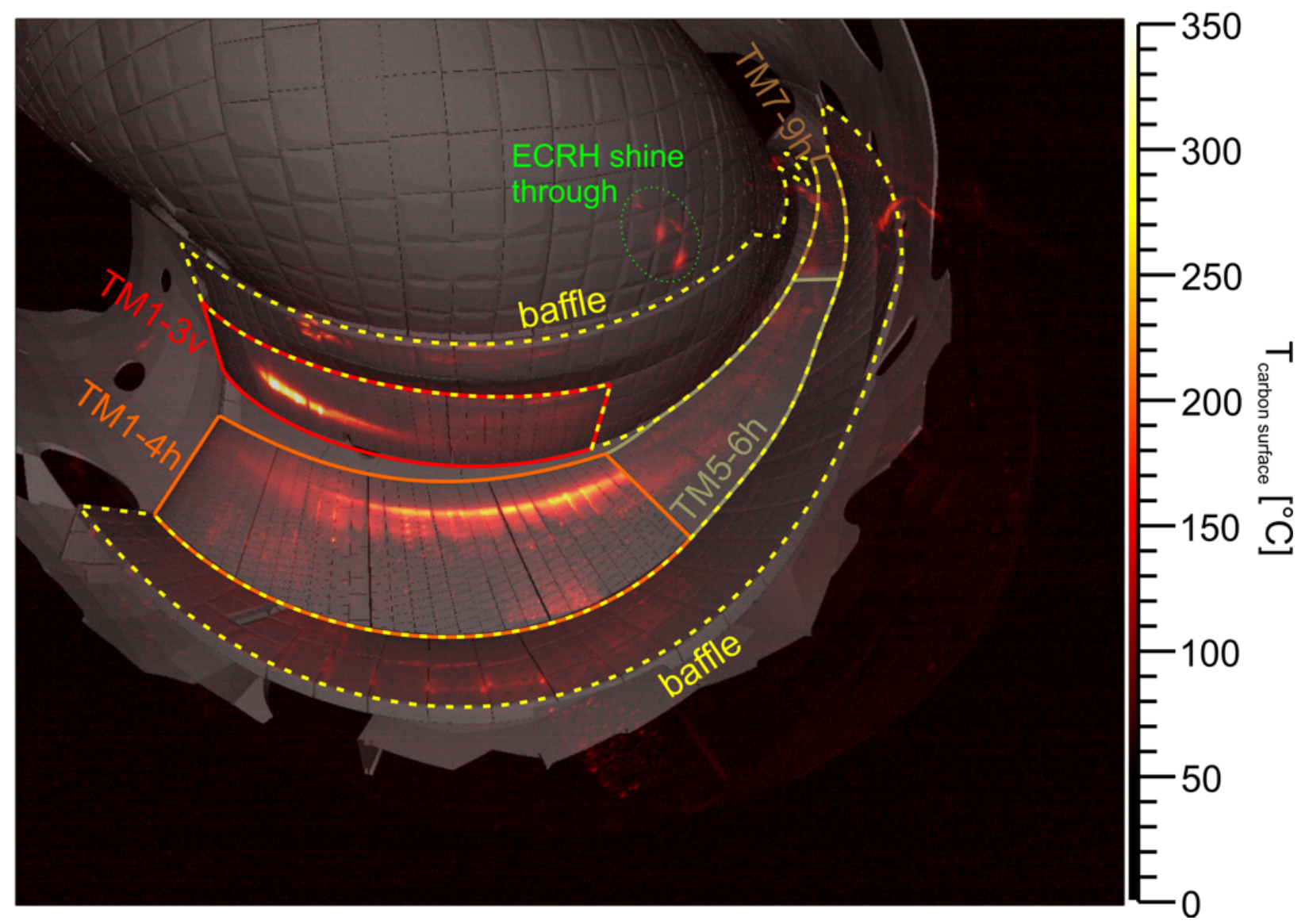

FIG. 6. Infrared image of a plasma vessel taken with a prototype IR/VIS endoscope. Image has been overlaid with the CAD model of the vessel. The CAD model is adapted to match the optical distortion of the endoscope's optics. Colors indicate carbon surface temperature.

lenses towards imaging cameras, allowing the observation of the divertor surface in infrared $(3-5 \mu \mathrm{m})$ and visible $(350-800 \mathrm{~nm})$ light. From the pinhole divertor light is directed via two mirrors (M1, M2 in Fig. 7) onto the off-axis Cassegrain optical system (M3, M4). The off-axis design helps avoiding diffraction due to central obscuration. From the M4 mirror, light passes through the window W2, where it is divided by the dichroic beam splitter (B1) into visible and infrared beams. After passing through a set of correcting lenses it is detected by the sensors in cameras (C1 and C2). Mirror M5 and window W1 provide an additional optical access to the divertor. By using M5 as a flip mirror the divertor can be illuminated for in-vessel inspection with a visible light source. In the other direction, simultaneously, divertor light can be imaged onto optical fiber rows connected to various spectrometers for edge and divertor plasma studies. 


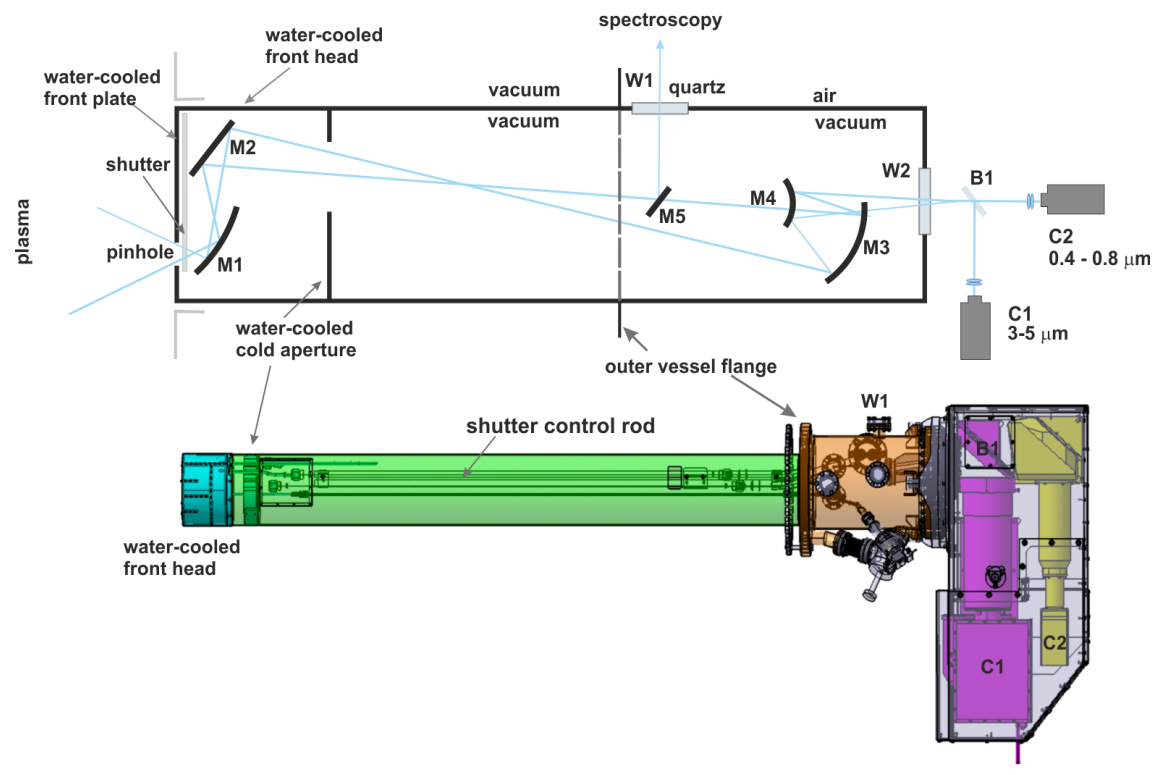

FIG. 7. (top) Functional sketch of the prototype IR/VIS endoscope used in OP1.2. (b) Actual CAD model snapshot of the prototype endoscope. M1-M2: aluminium front mirrors, M3-M4: offaxis Casegrain mirrors (Zerodur with IR/VIS coating), B1 - dichroic beam splitter, C1: infrared camera, C2- visible camera, M5,W1 - mirror and quartz window guiding divertor light towards spectroscopic diagnostic.

The theoretical optical performance of the prototype endoscope (used in OP1.2) is presented in Fig. 8. For the optical design, the divertor area is simplified into three flat rectangular areas corresponding to target areas TM1-3v, TM1-4h and TM7-9h. The optical performance requirement is specified at the corners of these areas as a minimum contrast of $15 \%$ MTF (modular transfer function) for a line pair of 3 (TM1-3v) or 4 mm thickness under consideration of the inclination of the respective area relative to the incident angle. The performance of the system is close to sufficient for the standard divertor and would allow us to resolve in most cases power loads on each of the target elements. However, the high iota part (7-9h) with MTF of $15 \%$ being reached at line pair width of $10-20 \mathrm{~mm}$ is far from sufficient. Nevertheless, a prototype endoscope provides useful information and good quality of infrared data for the standard divertor part (TM1-3v, TM1-4h) in OP1.2.

Choosing a pinhole instead of a window means that a constant influx of plasma impurities will enter into the endoscope, which will result in a slow degradation of the optical system transmission due to deposition of a:C-H layers (especially on the surface of the first mirror M1). The use of a pinhole is complicated further by having to water cool the plasma facing 

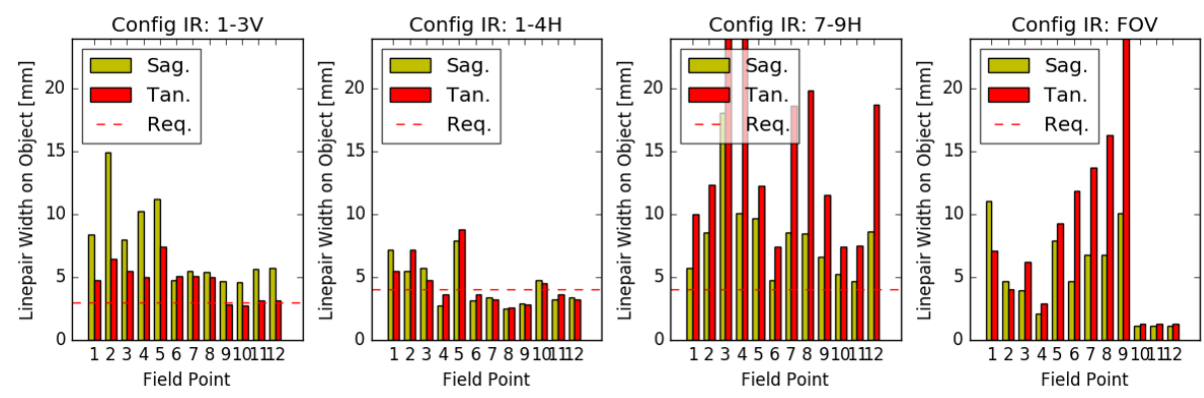

FIG. 8. Optical performance of prototype endoscope for different parts of the divertor. Bars denote line pair width $[\mathrm{mm}]$ on object side at which MTF is at least $15 \%$ (yellow in sagital direction, red in tangential direction). Red dashed line indicates a optical resolution to observe each of the blocks forming target elements (see FIG. 2). Graphs present the data for different parts of the image. From left: target area TM1-3v, target area TM1-4h, target area TM7-9h and corner points of field of view (see Fig. 1b).

front of the immersion tube in order to keep temperatures on endoscope front head and the cold aperture below $150^{\circ} \mathrm{C}$. Nevertheless, the choice of a pinhole over a front window seems to be justified, as loss of reflectivity of a mirror is usually lower than loss in transmission of a window given the same coating. Additionally several mitigation measures are applied in order to minimize this issue. An important element is a shutter, which closes the pinhole directly after the discharges to reduce carbon deposition on the mirrors ${ }^{18}$ and is closed during plasma vessel conditioning, i.e. glow discharge cleaning and boronisation. Inside the shutter a ceramic heater (made of $\mathrm{Si}_{3} \mathrm{~N}_{4}$ ) is installed for regular transmission monitoring. Also, as most of the incoming impurities will be deposited on the first mirror (M1), it is equipped with a heater mounted on the mirror back side. According to Maruyama et al. ${ }^{19}$ heating M1 up to $400^{\circ} \mathrm{C}$ will allow removal of most of the deposited amorphous hydrocarbon (a:C-H) layers. Heater tests and an evaluation of its effectiveness to remove surface layers will be performed after the end of this campaign.

One of the issues to be considered when designing a steady state diagnostic operating at W7-X (OP2) is absorption of ECRH stray radiation. In X2 mode ECRH power ${ }^{3}$, this radiation is well absorbed by the plasma for densities below the cut-off density of $1.25 \cdot 10^{20}$ $\mathrm{m}^{-3}$. However, for higher densities, the non-absorbed power fraction can be on the order of $20 \%$ or higher, which gives ca. $8 \mathrm{~W}$ of the ECRH stray radiation entering through a pinhole 

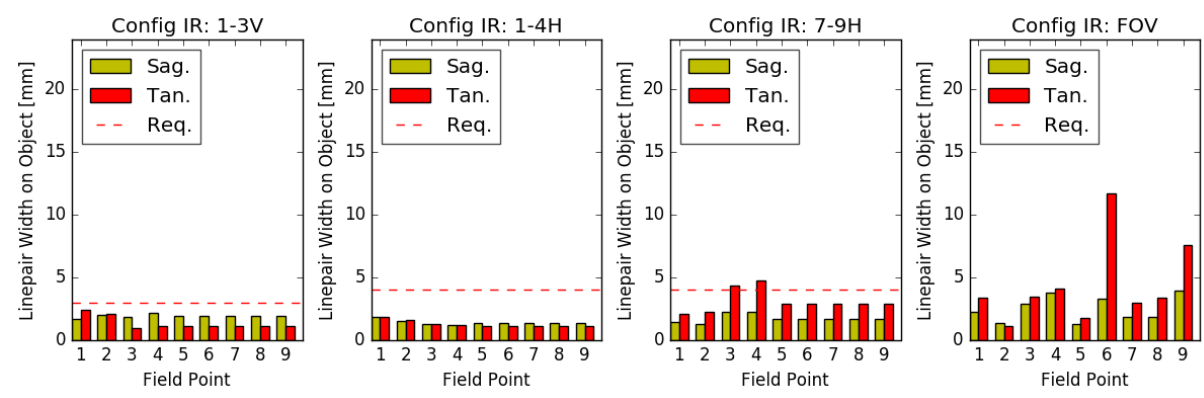

FIG. 9. Optical performance of a new on-axis Cassegrain endoscope designed for OP2. Bars denote line pair width $[\mathrm{mm}]$ on object side at which MTF is at least $15 \%$ Graphs present the data for different parts of the image. From left: target area TM1-3v, target area TM1-4h, target area TM7-9h and corner points of field of view (see Fig. 1b).

with diameter of $10 \mathrm{~mm}$. As the absorption coefficients of stainless steel is only on the order of a few percent, the rest of the stray radiation will form an equilibrium inside the endoscope, as it will undergo a very large number of reflections from the surface until it is finally absorbed. Therefore, any element with an absorption coefficient higher than stainless steel will receive a significant fraction of the power that enters the endoscope. It can lead to significant thermal loads on strongly absorbing components (e.g. ceramic) during a 30 minutes long discharge. To minimize that problem a water-cooled element, so-called "cold aperture", coated with $\mathrm{Al} 2 \mathrm{O} 3 / \mathrm{TiO} 2$ (with absorption coefficient of $70 \%$ for ECRH stray radiation) is installed inside the endoscope (see Fig. 7).

A typical image of the W7-X vessel taken with the prototype endoscope is shown in Fig. 6. The thermal image taken by the infrared camera is overlaid with the CAD model of the vessel. Two strike lines are positioned on the standard divertor in the areas TM1-4h and TM1-3v. The intensity indicates surface temperature. The image is corrected taking into account optical deformations (see Sec. III B). The resolution in the TM1-4h target area is ca. $5 \mathrm{~mm}$, which would be sufficient for a protection of high heat flux divertor. However in the high iota area (TM7-9h) the optical resolution on the divertor surface is ca. 10-20 $\mathrm{mm}$, which is below the requirements. Therefore, the prototype design had to be optimized before starting to build the required mini series of ten endoscope systems.

The optical performance requirement for the revised design has been checked and sharp- 


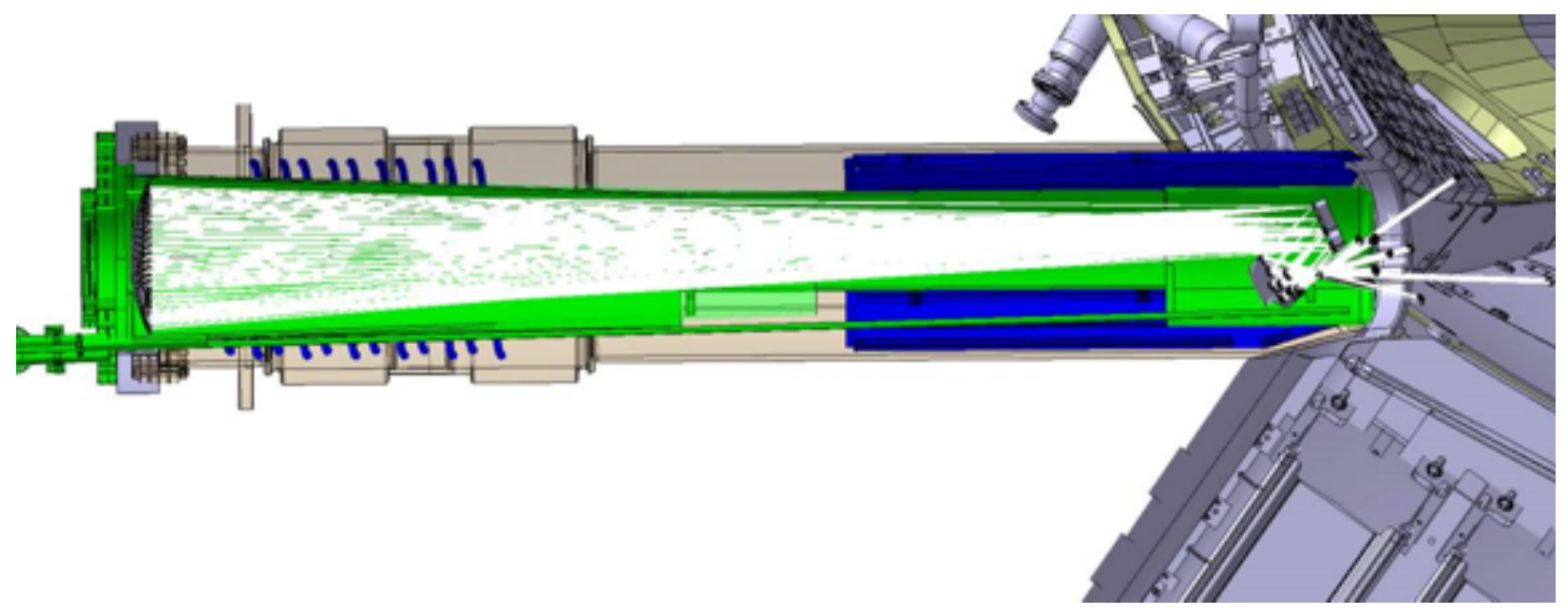

FIG. 10. Snapshot of CAD design of the new endoscope installed in the AEF port of W7-X.

ened such that each target element $(25 \times 55 \mathrm{~mm})$ should be resolved with at least three pixels in each direction and that the temperature reading of the middle pixel is not biased by the hot leading edges. After evaluation of 3 alternatives, a system similar to the existing design was selected. Each system consists of a water cooled vacuum plug-in collecting the plasma light via a pinhole and two front mirrors (M1 curved, M2 flat) and guiding the light to the rear end via an axial symmetric telescope mirror system (M3 and M4 both Q-aspheric shaped) and a sapphire vacuum window. At the air side, a beam splitter splits the plasma light into an IR (3-5 $\mu \mathrm{m})$ and visible (300-800 $\mathrm{nm})$ light channel and each channel passes through a series of lenses and fold mirrors before being captured on a IR and VIS cameras, respectively.

Key differences between the existing (OP1.2) and new (OP2) design are the use of a larger pinhole, an axial symmetric telescope instead of an off axis telescope with an additional aperture, and a cooled IR camera with a focal ratio of $\mathrm{f} / 1.2$ instead of $\mathrm{f} / 2$. As a result, the predicted line pair width at $15 \%$ MTF is a factor 2-3 finer at TM1-3v and TM1-4h and a factor 4-5 finer at TM7-9h than for the prototype design, compare Fig. 8 and Fig. 9. In addition, in the revised mechanical design, the optical components are thermally and mechanically isolated from the water cooled head to prevent imposed deformations that otherwise would harm the optical performance. 


\section{Scraper element observation}

Another object for infrared monitoring has been added with the installation of a divertor extension in two modules, so called scraper elements $(\mathrm{SE})^{20}$. In $\mathrm{OP} 1.2 \mathrm{~b}$, a separate imaging system $^{21}$, will be used with a view towards the upper divertor and its scraper element in Module 5. This diagnostic combines infrared images (up to $900 \mathrm{~Hz}$ framing rate with 200x200 sub-windowing, capable of measuring temperatures to $1500{ }^{\circ} \mathrm{C}$ ), interference filtered visible images with $1.5 \mathrm{~mm}$ spatial resolution, and a spectrometer averaging over the imaging spatial region, with $0.5 \mathrm{~nm}$ resolution from 200-1100 nm. A circular field of view across $1000 \mathrm{~mm}$, includes most of the $700 \times 400 \mathrm{~mm}$ graphite scraper element. It is made possible by viewing through a shuttered 4 -mm diameter pinhole, via two in-vacuum aluminum $90^{\circ}$ off-axis parabolic mirrors. The light is then relayed out through an uncoated sapphire vacuum window (137 mm diameter), where it is split into visible and IR paths using a 150 mm diameter silicon beam-splitter, and passed to the cameras. The metal optics in the AEK51 port provide a nearly aberration free image.

\section{SOFTWARE DEVELOPMENT}

\section{A. Data acquisition}

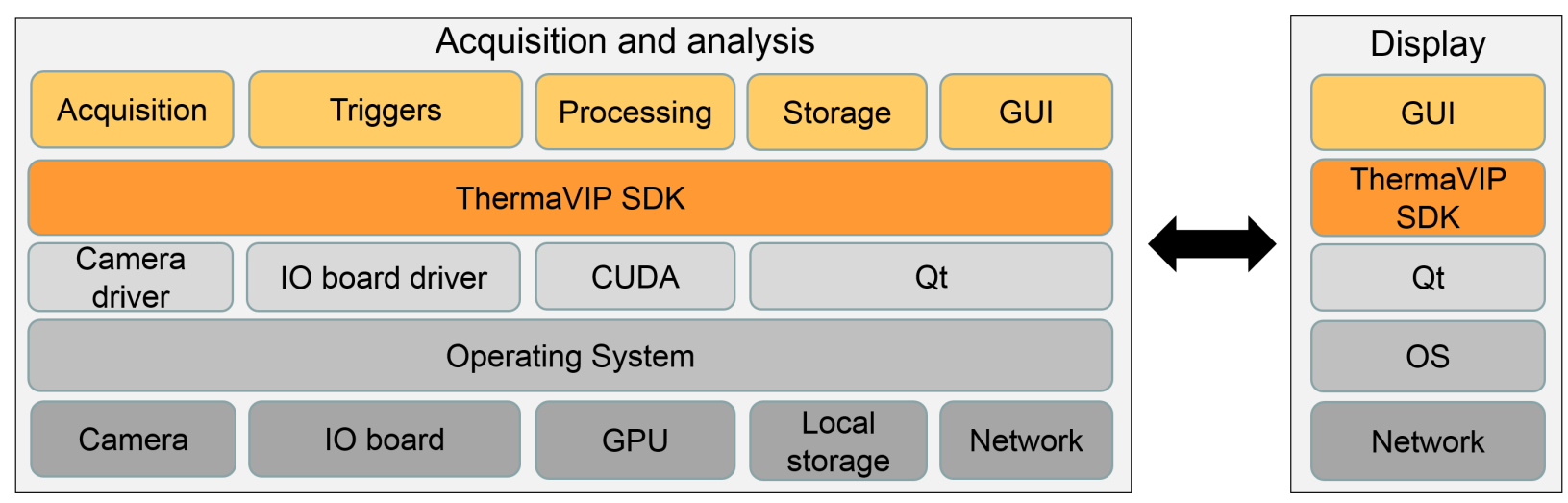

FIG. 11. The software is a distributed system of 10 computers for acquisition and analysis, and one central computer for visualization and control. The software is based on the ThermaVIP SDK, an open source $\mathrm{C}++$ framework for real-time and offline data analysis.

The control software is a distributed system of 10 acquisition computers and one central 
server computer in the control room. It consists of a set of plugins that extend the functionalities of ThermaVIP 22 , an open source $\mathrm{C}++$ framework for real-time and offline data analysis developed at CEA/ThermaDIAG. This framework is based at the same time on the CUDA library for GPU computation and the Qt library for operating system portability (see Fig. 11). The acquisition computers acquire the video streams from the thermographic cameras and they send them in real-time to the control room for visualization and control of the system.

\section{B. Lens distortion correction}

Finding a good camera model and correcting the lens distortion for IR imaging quality is really important, especially for cameras with wide angle lenses, characterized by large image distortions. The mathematical model most commonly used for camera calibration is the pinhole camera model, in which the camera aperture is described approximately as a point ${ }^{23}$. This model describes the relationship between each 3D point and its image projection in the image plane, by considering the position and orientation of the camera (extrinsic parameters), the position of the principal points and focal lengths in pixels (intrinsic parameters), and the lens distortion coefficients.

A quite accurate estimation of the intrinsic parameters and lens distortion coefficients of the model of the immersion tubes has been obtained ${ }^{14}$ with multiple views of a chessboardlike object, by using the algorithm $\mathrm{in}^{24}$. The algorithm consists of a closed-form solution, followed by a nonlinear refinement based on the maximum likelihood criterion.

The model has been then completed by adding the extrinsic parameters, i.e. position and orientation of the camera. These parameters have been initialized by the nominal position and orientation of the immersion tubes, and refined with a nonlinear least-square criterion based on some control points and a simplified CAD model of the PFCs. The undistorted images are presented in Fig. 5 and Fig. 6, which have been achieved by adapting the projection

of the vessel CAD model to the optically distorted (fish eye distortion) image ${ }^{14}$. 


\section{Real-time protection}

During the high heat flux operation of Wendelstein 7-X from OP2 onwards, the real-time protection of the plasma facing components will be mandatory during plasma operation to avoid degradation of critical plasma facing components. The endoscopes will be used to detect overheated areas on the divertor surface (with temperature above $1000^{\circ} \mathrm{C}$ ), and failures in thermal connection of the $\mathrm{CFC}$ elements of the divertor to the cooling structure and symmetry and structure of the power loads in the 3D environment of Wendelstein 7-X. As the discharge will be up to 30 minutes long, an excessive amount of data (up to 2.4 $\mathrm{TB} /$ day) is expected to be submitted to the image processing routines. Therefore, all the data storage, real-time analysis and a major fraction of the post-processing needs to be fully automatized. The data processing is performed with the software packages discussed in Sec. III A, which rely on intelligent vision system concepts. The strategy of analyzing the infrared images of W7-X divertor is composed of two main tasks: hot spot detection

and thermal event recognition ${ }^{25,26}$. Hot spots on the carbon plasma facing components may appear for a number of reasons:

- Overheating due to excessive power loads. As mentioned above, the target elements can withstand heat fluxes up to a certain level. For instance, baffle structures can cope with steady state heat flux density of up to $0.5 \mathrm{MW} / \mathrm{m}^{2}$. It may happen that due to a change of plasma configuration or bootstrap currents additional power loads reach baffle elements.

- Delaminated carbon tiles will lose their thermal connection to cooling structures of the divertor and therefore will not be able to efficiently remove the power stored in their bulk material. This will result in an elevated CFC temperature.

- Due to carbon erosion and re-deposition thin surface layers are formed on the plasma facing components during plasma operation ${ }^{27}$. Typically they have very a poor thermal connection to the bulk material and therefore heat up very fast and reach much higher temperatures than a virgin surface of the $\mathrm{PFCs}^{28}$.

- Localized fast particle losses from the neutral beam heating system (NBI) can also produce the hot $\operatorname{spots}^{29}$, the duration of the beam injection is up to $10 \mathrm{~s}$. First injections will be performed during second part of the OP1.2 campaign. 


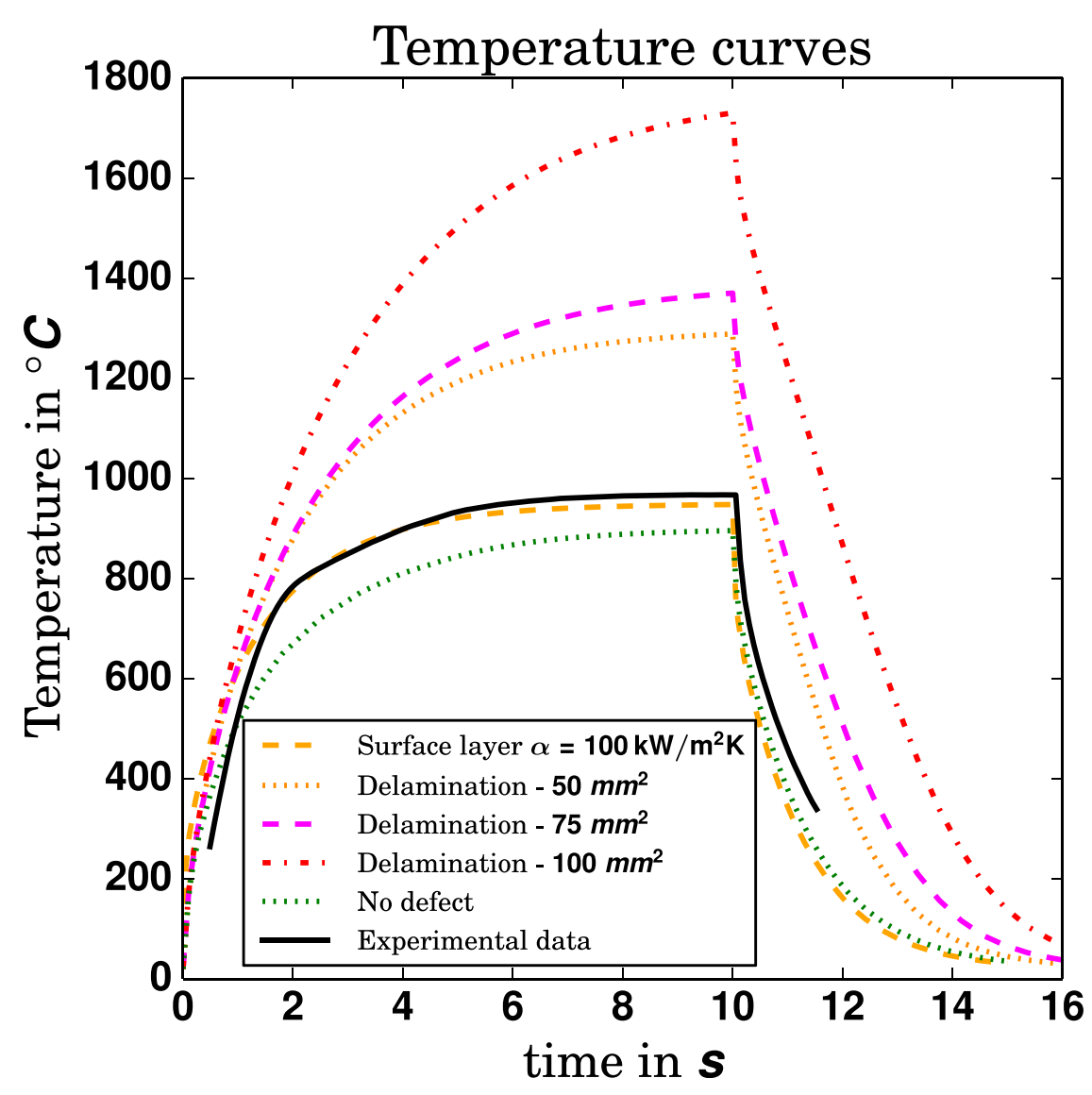

FIG. 12. (a) Surface temperature curves for tiles of different status. Data were gained by FEM calculations. Heating was switched off at $t_{\text {off }}=10 \mathrm{~s}$. The different transient behaviors for target tiles with a surface layer, delamination, or intact are visible. (b) Result of tests in experimental facility GLADIS. Reproduced from [Rodatos, et al., Rev. Sci. Instr., 872016 023506] with the permission of AIP Publishing.

- Dust particles formed inside the vessel tend to be redeposited during the discharge. As they are poorly thermally connected, they can become very bright.

The video streams are analyzed online by means of computer vision techniques to detect thermal events like hot-spots, strike-lines and other dangerous events. As discussed in Sec. III C hot-spots are classified between power overloads and surface layers to avoid false alarms. A scene model provides a pixel-wise information of the target elements, their material, emissivity and maximum allowed temperature. In future, a risk analysis will be performed on each detected thermal event taking into account the target information, the hot-spot size and temporal evolution. If the temperature reaches a certain threshold an 
alarm is generated to the interlock system which connects with the central Safety System of the device. All the detected thermal events and alarms are shown in the control room in the form of a thermal map and they are stored in a SQL database for further offline analysis.

An intelligent real time protection system, which is based on the imaging of the PFCs with the infrared cameras must be able to classify the events based on their origin. At the moment the algorithms are being developed to aid such classification ${ }^{22,25,26}$. One of the methods is to apply transient power fluxes and to observe the time evolution of the surface temperature (see Fig. 12). Due to vastly different thermal conductivity and thermal capacity, a delaminated tile will show a measurably different temporal behavior of surface temperature than a healthy tile, or one with surface layers. The results of Fig. 12(a) have been modeled with the finite element method code ANSYS ${ }^{25}$. Results from four studied cases are presented, where time traces are shown for a load scenario where $10 \mathrm{MW} / \mathrm{m}^{2}$ have been applied for 10 seconds. The reference case is shown in green, an intact tile, where after about $5 \mathrm{~s}$, a thermal equilibrium at $\mathrm{T} \approx 700^{\circ} \mathrm{C}$ is reached. The intact tiles show the smallest amplitude of temperature change due to incoming power flux. If a target element has a corner delamination of the graphite from the underlying cooling structure with a surface area of $58 \mathrm{~mm}^{2}$ the difference from the intact tile is rather small. However, with increasing delamination area, the time to reach thermal equilibrium is longer and it is established at much higher surface temperatures. Also the cooling of the target element after switching off the heat loads needs more time. The fourth case is a tile with a surface layers made from a:C-H co-deposits with thickness on the order of a few $\mu \mathrm{m}$. Here, in contrast, a very fast thermal response to the applied power loads is observed. The reason are low thermal capacity of the co-deposited layer and poor thermal connection to underlying bulk material. These different thermal reactions of the virgin tiles, the delaminated tiles and those with co-deposits will be used at W7-X to classify the observed areas with elevated temperatures by introducing a so-called $\tau$ criterion ${ }^{25}$ :

$$
\tau=\frac{T(t)}{\partial_{t} T(t)}
$$

This algorithm together with algorithms to detect hot spots, their clustering and surface layer detection were adapted to work in near real-time at W7-X. The experiments performed in the GLADIS facility on pre-series, water-cooled tiles were able to detect $\mathrm{CFC} / \mathrm{Cu}$ 
delaminations $^{26}$. In the present campaign surface layers and hot spots can be detected in real-time. The next step will be to hardwire a connection to the W7-X fast interlock system in order to trigger alarms. This will be tested in the following months and it is planned to be operating at the beginning of OP2. More sophisticated tools like real-time strike line tracking and control with help of deep learning methods will be developed in the course of OP2.

In order to accommodate changes in the emissivity of a divertor graphite surface a comparison with measurements of the divertor calorimetry will be performed. In thermal equilibrium (indicated by the calorimetry) all target elements should have the same surface temperature. A difference in IR measurements indicates different emissivity.

\section{SUMMARY}

Wendelstein 7-X is equipped with a number of infrared diagnostics in order to protect plasma facing components in three dimensional environment of a stellarator experiment. Several challenges have been tackled while developing the diagnostic. First and foremost to design optics allowing high resolution IR observation with a wide angle field of view. The endoscopes have to operate in quasi steady state (up to 30 minutes), i.e. be resistant to ECRH stray and plasma thermal radiation. Additionally, it is required to process in near

real time as well offline large amount of data. One of the highlights of the W7-X infrared protection system is the application of novel techniques for image and data processing.

\section{ACKNOWLEDGMENTS}

This work has been carried out within the framework of the EUROfusion Consortium and has received funding from the Euratom research and training programme 2014-2018 under grant agreement No 633053. The views and opinions expressed herein do not necessarily reflect those of the European Commission. 


\section{REFERENCES}

\section{REFERENCES}

${ }^{1}$ WOLF, R., ALI, A., ALONSO, A., et al., Nuclear Fusion 57 (2017).

${ }^{2}$ FEDERICI, G., SKINNER, C., BROOKS, J., et al., Nuclear Fusion 41 (2001) 1967.

${ }^{3}$ ERCKMANN, V., BRAUne, H., LAQUA, H. P., et al., The $10 \mathrm{MW}$ ECRH and CD System for W7-X, in AIP Conference Proceedings, volume 691, pp. 213-223, AIP, 2003.

${ }^{4}$ GRIGULL, P., MCCORMICK, K., RENNER, H., et al., Fusion Engineering and Design 66-68 (2003) 49.

${ }^{5}$ BOSCARY, J., STADLER, R., PEACOCK, A., et al., Fusion Engineering and Design 86 (2011) 572 .

${ }^{6}$ Sunn Pedersen, T., ANDREEVA, T., BOSCH, H.-S., et al., Nuclear Fusion 55 (2015) 126001.

${ }^{7}$ KÖNIG, R., BIEL, W., BIEDERMANN, C., et al., Review of Scientific Instruments 85 (2014) 11D818.

${ }^{8}$ KRYCHOWIAK, M., ADNAN, A., ALONSO, A., et al., Review of Scientific Instruments 87 (2016) 11D304.

${ }^{9}$ HATHIRAMANI, D., ALI, A., ANDA, G., et al., Fusion Engineering and Design (2018).

${ }^{10}$ GREUNER, H., BÖSWIRTH, B., BOSCARY, J., et al., Fusion Engineering and Design 84 (2009) 848.

${ }^{11}$ GREUNER, H., BOESWIRTH, B., BOSCARY, J., and MCNEELY, P., Journal of Nuclear Materials 367-370 (2007) 1444.

${ }^{12}$ SIEGLIN, B., FAITSCH, M., HERRMANN, A., et al., Review of Scientific Instruments 86 (2015) 113502.

${ }^{13}$ WURden, G., BIEDERmann, C., EFFEnBERG, F., et al., Nuclear Fusion 57 (2017) 056036 .

${ }^{14}$ PISANO, F., CANNAS, B., JAKUBOWSKI, M., NIEMANN, H., and Puig Sitjes, A., to be published

${ }^{15}$ CANTARINI, J., HILDEBRANDT, D., KÖNIG, R., et al., Review of Scientific Instruments 79 (2008) 10F513. 
${ }^{16}$ BALBOA, I., ARNOUX, G., EICH, T., et al., Review of Scientific Instruments 83 (2012) $10 \mathrm{D} 530$.

${ }^{17}$ CHAUVIN, D., JAKUBOWSKI, M., JENZSCH, H., GAMRADT, M., and LORENZ, A., Design and manufacturing progress of IRVIS endoscopes prototypes for W7-X divertor temperature monitoring Preprint of Paper to be submitted for publication in, in Proceedings of 29th Symposium on Fusion Technology (SOFT 2016), 2016.

${ }^{18}$ VON KEUDELL, A., HOPF, C., SCHWARZ-SELINGER, T., and JACOB, W., Nuclear Fusion 39 (1999) 1451.

${ }^{19}$ MARUYAMA, K., JACOB, W., and ROTH, J., Journal of Nuclear Materials 264 (1999) 56.

${ }^{20}$ HÖLBE, H., Sunn Pedersen, T., GEIGER, J., et al., Nuclear Fusion 56 (2016) 026015.

${ }^{21}$ WURDEN, G. A., FELLINGER, J., BIEDERMANN, C., et al., Review of Scientific Instruments (2018).

${ }^{22}$ Puig Sitjes, A., JAKUBOWSKI, M., ALI, A., et al., Fusion Science and Technology (2017) 1.

${ }^{23}$ FORSYTH, D. and PONCE, J., Computer vision : a modern approach, Pearson, 2012.

${ }^{24}$ ZHANG, Z., IEEE Transactions on Pattern Analysis and Machine Intelligence 22 (2000) 1330 .

${ }^{25}$ RODATOS, A., GREUNER, H., JAKUBOWSKI, M. W., et al., Review of Scientific Instruments 87 (2016) 023506.

${ }^{26}$ ALI, A., JAKUBOWSKI, M., GREUNER, H., et al., Physica Scripta T170 (2017) 014074.

${ }^{27}$ LIKONEN, J., COAD, J., VAINONEN-AHLGREN, E., et al., Journal of Nuclear Materials 363-365 (2007) 190.

${ }^{28}$ HILDEBRAnDT, D., GADELMEIER, F., GRIGUlL, P., et al., Thermographic observation of the divertor target plates in the stellarators W7-AS and W7-X, in Journal of Nuclear Materials, volume 313-316, pp. 738-742, 2003.

${ }^{29}$ WERNER, A., WELlER, A., DARROW, D. S., and TEAM, T. W.-A., Review of Scientific Instruments 72 (2001) 780. 